\title{
Comparative Data Concerning the Incidence of Tumors in Dogs in a Period of Ten Years in Athens (Greece) and Cluj-Napoca (Romania)
}

\author{
Adrian Florin GAL, Anna ANDRIOPOULOU, Viorel MICLĂUŞ, Flaviu TǍBĂRAN, Marian TAULESCU, \\ Andras NAGY, Vasile RUS*, Roxana CORA, Raluca VIDRIGHINESCU, Cornel CĂTOI \\ Faculty of Veterinary Medicine, University of Agricultural Sciences and Veterinary Medicine, 3-5 Mănăştur \\ Street, 3400 Cluj-Napoca, Romania. \\ *Corresponding author: vasilerus2002@yahoo.com
}

Bulletin UASVM Veterinary Medicine 72(2) / 2015,

Print ISSN 1843-5270; Electronic ISSN 1843-5378

DOI:10.15835/buasvmcn-vm: 11538

\begin{abstract}
Epidemiological studies in domestic animal populations can deliver useful details concerning the incidence of neoplastic lesions, which may aid in the identification of risk factors. In the proposed study, we considered the incidence and prevalence of tumors in dogs in Voula (a suburb area of Athens, Greece) and Cluj-Napoca (Romania) for a period of 10 years. The obtained data were compared in order to observe the main differences in the suggested regions. The data were collected from the records of Pathology Department (Faculty of Veterinary Medicine, Cluj-Napoca, Romania) and a hospital in Voula (a suburb area of Athens, Greece), respectively, in the last decade. Several differences were observed between Voula (Athens) and Cluj regions concerning tumor occurrence. The incidence of mammary gland neoplasm in Cluj County was two times higher compared to the findings from Voula (Athens). Furthermore, the occurrence of skin neoplasms was half in Cluj compared to the records in Greece, where skin neoplasms were the most prevalent tumors. Another difference was the incidence of tumors in the lymphatic system which was very low in Cluj (approximately six times less) than in Voula (Athens). Concerning the highest tumoral prevalence in various dog breeds, the most affected were Mongrel and German shepherd (somehow similar with the results obtained in Cluj County). The incidence of cancer in dog fluctuates according to the investigated area, suggesting that determining environmental risk factors may contribute to the neoplastic lesion development.
\end{abstract}

Keywords: cat, dog, incidence, prevalence, tumor.

\section{INTRODUCTION}

Cancer is one of the most important disease that affects humans but also companion animals. In 2008, 7.6 million cancer deaths were reported in humans while by 2030 this number is expected to be over 13.2 million even though the diagnosis and treatment of cancer have been radically improved (American Cancer Society, 2011).

Cancer is considered the main cause of death in pet animals (Bonnett et al., 2005) and the second cause of death in humans (Siegel et al.,
2013), while its frequency in dogs is two times higher than that of humans. Many types of cancer in companion animals have similar behavior, pathologic expression and risk factors with that of humans due to presence of carcinogens in the environment such as tobacco smoke, pesticides or other (Rungsipipat et al., 2003).

However, it has been very difficult to estimate the morbidity and mortality rate of cancer in animals. In human medicine, cancer cases have been registered with all the epidemiological data 
and studied since 1940s, whereas in veterinary medicine cancer cases have been recorded in smaller numbers, in a non-organized manner and there is very little information nowadays on the occurrence of different types of neoplasms in companion animals. Cancer registries provide information regarding the risk assessment for each case, the evaluation of incidence, epidemiological data, treatment options and the identification of risk factors. Therefore it is very important to keep records of all the epidemiological information not only for control and treatment of animals but also these data can be used as a warning system for humans since both animals and humans are exposed to similar environmental risk factors (Vascellari et al., 2009; Andriopoulou, 2015).

Epidemiological studies are very important in determining risk factors that may be involved in the initiation and progression of tumors. The most common risk factors involved are environmental agents, which together with other factors (e.g., genetic factors, viral particles) may promote tumorigenesis (Pershagen and Simonato, 1993; Hemminki and Pershagen, 1994; Cohen, 2000; Petrauskaite et al., 2002; Boffetta and Nyberg, 2003). According to the statistics, dogs get cancer at roughly the same rate as humans, while cats get fewer cancers (Rungsipipat et al., 2003; Vascellari et al., 2009; Popa, 2014). Because dogs are the one domestic animal that most closely shares the human environment, determining environmental risk factors for canine tumors may contribute to identification and understanding of such risk factors for similar tumors in humans.

Based on the above, this study will provide comparative information concerning the incidence and prevalence of tumors in dogs in Voula (a suburb area of Athens, Greece) and Cluj-Napoca (Romania) for a period of 10 years. The epidemiological data for all animals were recorded. The classification of tumors was based on histopathological diagnosis and then was categorized in different regions, age and sex.

\section{MATERIALS AND METHODS}

In the present study we assessed the incidence and prevalence of tumors in dogs during a period of ten years, in the Department of Pathology (FMV Cluj-Napoca, Romania) and in the region of Voula - Athens (Greece), respectively. In both regions, the records of the Department of Veterinary
Pathology Cluj-Napoca (in the period 2004-2013), and the documentation of an animal clinic in Voula - Athens (in the period 2005 - 2014) were used. All of the clinical cases of neoplasms during the suggested period were admitted in the animal clinic in the region of Voula, Athens. From each case, a biopsy sample was sent to Department of Pathology, Faculty of Veterinary Medicine, Aristotle University of Thessaloniki for histopathological diagnosis. However, concerning the records of the Department of Veterinary Pathology Cluj-Napoca (Romania), the material included corpses and biopsies brought for histological and/or necropsy examination.

Furthermore, all types of tumors that affected both skin and tissues of dogs were analyzed. Also, more information about each animal during this period was recorded by inquiring the owner during anamnesis, such as reproductive status of the animals whether the animal was neutered or intact.

All of the data previously mentioned were recorded in a table for every animal during the period of ten years. This table contains information for each case separately including the age, neutering status, breed, sex, body condition score, the anatomical location of the tumor, the histopathological diagnosis, type of tumor according to their nature (malignant or benign), the region of metastasis if occurred and finally cause of death if it was due to tumor or not. Moreover, the population of animals was assorted in several age categories (i.e., young subjects, middle age subjects and old subjects).

\section{RESULTS AND DISCUSSION}

The main pet species frequently diagnosed with neoplastic lesions are dogs and cats. From the two species, dogs appear to develop more often tumoral masses, aspect that was also observed in our investigation. Accordingly, from the total number of cases recorded in Athens, 84\% cases were recorded in dogs and the rest in cats (16\%). Comparatively, in Cluj County about $88.09 \%$ of tumor cases were recorded in dogs and $11.91 \%$ in cats. As observed similar data were noticed concerning the occurrence and distribution of tumor cases in the two species.

In Athens, the tumor incidence had an increasing tendency in the very last years, with highest percentages in 2011, 2013 and 2014. A 
similar pattern was noticed in Cluj County too (Fig. 1 and 2), with the highest levels in 2011 and 2013.

In Athens, the number of neoplasms that occurred in canine females corresponds to $40.2 \%$ and the tumor cases recorded in males was $59.8 \%$. As a comparison, in Cluj County about $61.54 \%$ of tumors were recorded in female dogs and $38.45 \%$ in male dogs. As presented above, the prevalence of neoplasms in the suggested regions was different with reference to the sexual category. A higher percentage of tumors occurred in males in Athens and in females in Cluj County.

The distribution of tumors in female dogs included: $21.6 \%$ of cases were carcinomas, $15.7 \%$ of cases were histiocytomas, $9.8 \%$ of cases were fibromas. Carcinomas comprise various types of neoplasms such as squamous cell carcinoma, solid and complex mammary carcinoma, tubulopapillary carcinoma of mammary gland, epithelial transitional carcinoma, broncholaveolar carcinoma, basosquamous carcinoma, basal cell carcinoma and hepatocellular carcinoma. The remaining cases were represented by adenocarcinoma, adenoma and hemangiosarcoma with around 5.9\% in each; fibrosarcoma, lipoma, mast cell tumor and trichoblastoma with 3.9\% of cases in each; fibromyxoma, leiomyoma, lymphoma, melanoma, osteosarcoma, pappiloma, round cell tumor, sarcoma and trichoepithelioma with $2.0 \%$ in each.

In the study conducted in Cluj county, the most prevalent types of tumors affecting female dogs were (in brief) carcinoma (47\%), fibroma/leiomyoma (6\%), adenocarcinoma (5.4\%), melanoma (5.22\%), adenoma (3.68\%), fibrosarcoma (3.68\%), lymphoma (3.68\%), bone tumors (3.48\%), sticker sarcoma (3.29\%), adipose

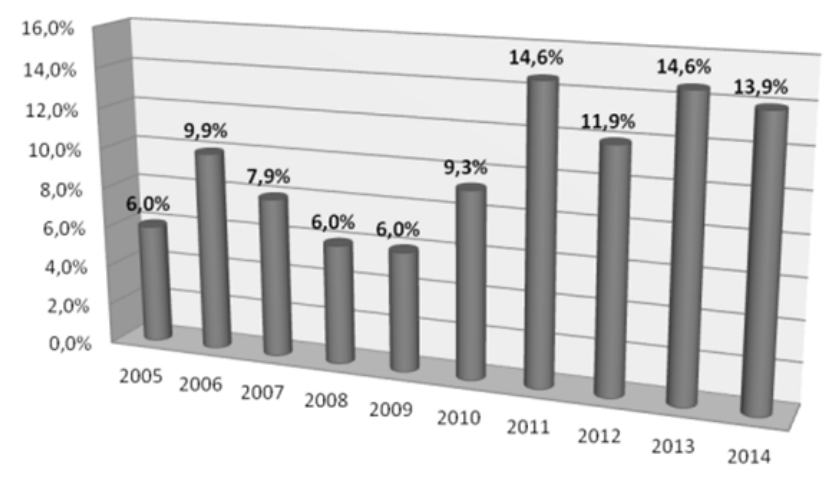

Fig. 1. Incidence of tumors per year in the investigated period in Athens, Greece. tissue tumor (3.09\%), mast cell tumor (3.09\%), vascular tumors (2.71\%), sarcoma $(2.13 \%)$, histiocytoma (1.93\%), papilloma (1.93\%), hair follicles tumors $(1.16 \%)$, chemodectoma $(0.77 \%)$, leiomyosarcoma $(0.77 \%)$ etc.

Concerning tumor incidence in female dogs in Athens and Cluj County (presented above), it can be observed that carcinomas were the most common type of tumor found in female dogs, but second in place was histiocytoma in Athens with $15.7 \%$ (versus $1.9 \%$ found in Cluj county) and then fibroma with $9.8 \%$ (which was the second most frequent tumor in Cluj County with 6\% cases). According to Kelsey et al. (1998), adenocarcinomas represented about $76 \%$ of breast cancers in dogs.

As regard the incidence of tumors in male dogs in Athens, the mast cell tumor and lymphoma had the highest incidence throughout the decade (i.e., $14.3 \%$ of cases in each). In second place, carcinomas occurred in $9.1 \%$ of cases, followed in the third place by epoulis, fibroma, hemangiosarcoma, hystiocytoma, seminoma, Sertoli cell tumor with $5.2 \%$ in each type of tumor. In fourth place we found cases of adenoma, lipoma, bone tumors (osteosarcoma and osteoma), papilloma and round cell tumors with $2.6 \%$ in each category. The remaining cases of $1.3 \%$ in each were represented by adenocarcinoma, angiolipoma, fibrolipoma, fibrosarcoma, hemangioma, melanocytoma, meningioma, trichoblastoma and vascular hamartoma.

In Cluj County, however, the most common type of tumor encountered in male dogs was the mast cell tumor $(11.5 \%)$, followed by vascular tumors (11.15\%), carcinoma (10.22\%), papilloma/ fibropapilloma (9.6\%), melanoma (7.43\%), Sertoli cell tumor (7.12\%), adenoma/fibroadenoma

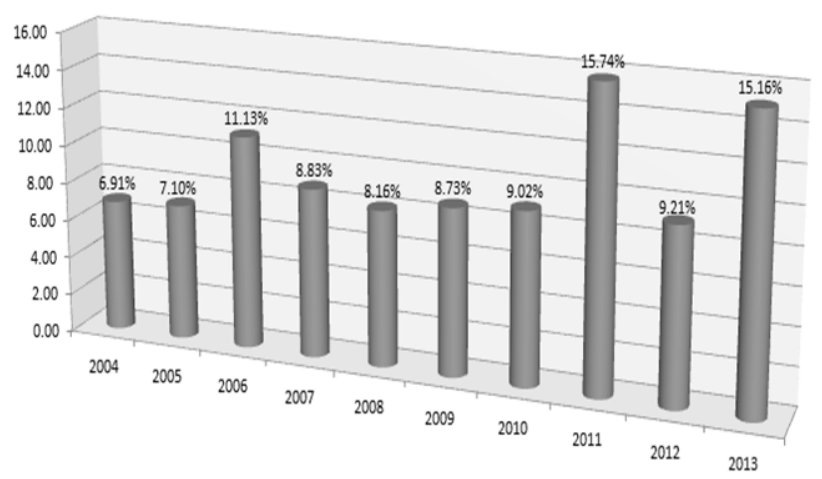

Fig. 2. Incidence of tumors per year in Cluj County, Romania. 
(5.26\%), lymphoma (4.95\%), histiocytoma $(4.95 \%)$, bone tumors $(4.02 \%)$, seminoma (3.41\%), sarcoma $(3.10 \%)$, hair follicles tumor (3.10\%), adenocarcinoma $(2.79 \%)$, fibrosarcoma (2.79\%), sticker sarcoma (2.17\%), adipose tissue tumor $(2.17 \%)$, epithelioma (2.10\%), fibroma/ leiomyoma $(0.93 \%)$ etc.

As a comparison, while in Athens the mast cell tumor and lymphoma (14.3\% in each) were the most prevalent types of neoplasms in male dogs, in Cluj County the mast cell tumor and vascular tumor $(11.5 \%)$ prevailed. On the contrary, only $4.9 \%$ cases of lymphoma in male dogs were reported in Cluj. Some differences existed between the prevalence of cases with Sertoli cell tumor and seminoma (i.e., a slightly higher incidence of Sertoli cell tumor in Cluj County $-7.12 \%$ versus $5.2 \%$ in Athens; a lower occurrence of seminoma in Cluj comparing to Athens - $3.4 \%$ versus $5.2 \%$ ). Another interesting finding after comparing the incidence of different types of tumors in male and female dogs was that the incidence of mast cell tumor in males was three to four times higher compared to female dogs, aspect that can be verified in both Athens and Cluj County.

Some types of tumors were more commonly diagnosed in female dogs and others in male dogs, as is the case of hepatoid gland tumors that occurs more often in males (Baba and Cătoi, 2007). Kelsey states that there is no sex predisposition in lymphoma (Kelsey et al., 1998). However, in our findings from Athens, it is noticed that the lymphoma cases were substantially higher in male dogs with $14.3 \%$, whereas in female dogs only $2.0 \%$ of cases occurred. On the contrary, in Cluj County we did not observed something similar.

The incidence of tumors according to regions and systems in dogs from Athens, in the first place was ranked the skin with $43.1 \%$, in second place was the mammary gland with $23.5 \%$ and in the third place were the lymphatic and reproductive systems with $7.8 \%$ in each. Then the oral cavity follows with $5.9 \%$ and the auricular region with $3.9 \%$. Finally, in the last place are the muscular, respiratory, skeletal and urinary systems with only $2.0 \%$ in each.

Concerning the Cluj County, the regions in which usually neoplasms developed in female dogs were the mammary gland (48.41\%), skin $(22.2 \%)$ and reproductive system $(11.2 \%)$. The next regions involved were the following: bones and joints (3.17\%), oral cavity (3.02\%), orbital region $(2.54 \%)$, alimentary tract $(2.06 \%)$, respiratory tract $(1.59 \%)$, lymph nodes $(1.27 \%)$, urinary tract $(0.79 \%)$, liver $(0.79 \%)$, endocrine $(0.63 \%)$ and other regions.

Generally, in both studies the regions that were mostly affected by neoplasms in female dogs were the mammary gland and the skin. After comparing these findings, it is observed that the highest incidence of tumor appeared in skin ( $43.1 \%$ in Athens vs. $22.2 \%$ in Cluj County), then in mammary gland $(23.5 \%$ in Athens vs. $48.1 \%$ in Cluj County) and finally the reproduction system (7.8\% in Athens vs. $11.2 \%$ in Cluj County). The incidence of tumor in the lymphatic system of female dogs in Cluj County was only $1.27 \%$ comparing to a much higher percent in Athens (7.8\%).

The occurrence of tumors in male dogs according to body regions and systems in Athens was as follows: skin with $42.1 \%$, lymphatic system with $18.4 \%$, reproductive system with $14.5 \%$, oral cavity follows with $7.9 \%$, the orbital region with $5.3 \%$, digestive and skeletal systems with $2.6 \%$ in each and finally with only $1.3 \%$ recorded in each follows the auricular region, mammary gland, nervous, urinary and respiratory systems.

In the epidemiological report realized in Cluj County, the most common regions and systems affecting male dogs were the skin (50.73\%), reproductive system (13.59\%), oral cavity $(8.25 \%)$, alimentary tract $(7.77 \%)$, orbital region (4.37\%), bones and joints $(4.13 \%)$, urinary tract $(2.91 \%)$, liver $(2.43 \%)$, respiratory tract $(1.94 \%)$, lymph nodes $(1.21 \%)$, endocrine $(0.49 \%)$, and other regions.

In both studies (from Athens and Cluj County) the most prevalent systems affected by tumors in male dogs involved the skin and the reproductive system with similar percentages. However, some differences were recorded in the digestive system (i.e., $7.7 \%$ in Cluj County vs. $2.6 \%$ in Athens). A major difference was noticed in the lymphatic system with $18.4 \%$ of tumor cases in Athens vs. only $1.2 \%$ in Cluj County.

As observed, some differences concerning the neoplasms prevalence/body regions existed between female and male dogs. The most common systems affected by tumors in male dogs were the skin, reproductive system and lymphatic system (the last only in Athens), whereas in female dogs it was the mammary gland and the 
skin. The reproductive system in male dogs was more frequently affected by tumors comparing to female dogs (mammary glands not included). There was no considerable difference between female and male dogs in the tumors developed in the oral cavity, the eye and the skeletal system. Finally, no differences were detected between female and male dogs in skin which is also stated by Kaldrymidou et al. (2002).

Brearley (1989) states that $50 \%$ of all neoplasms in female dogs are represented by tumors of the mammary gland, somehow similar with the results obtained in Cluj County but not in Athens (where the cutaneous tumors were almost the double of the mammary gland neoplasms). According to Bostock (1986a,b), the most common neoplasm found in dogs arises from the skin, which coincides with our results. The number of reported cases in skin was so high probably because it is easier for owners to observe them, but also due to higher exposure to various physical and chemical agents.

With reference to the incidence of tumors by age in Athens, the regions that were more affected in different age groups were: (a) 0-6.9 years old: skin $65 \%$, oral cavity $7.5 \%$, mammary gland $7.5 \%$, skeletal system and lymphatic system $5 \%$ in each, respiratory system $2.5 \%$, reproductive system $2.5 \%$, muscular system $2.5 \%$, orbital region $2.5 \%$; (b) 7-11.9 years old: skin 36.4\%, reproductive system $15.2 \%$, mammary gland $13.6 \%$, lymphatic system $13.6 \%$, oral cavity, auricular and orbital region $4.5 \%$ in each, digestive system $3 \%$, urinary tract $1.5 \%$, bone and joins $1.5 \%$, nervous system 1.5\%; (c) 12-16 years old: lymphatic system $33.3 \%$, reproductive system and skin $19 \%$ in each region, oral cavity $14.3 \%$, mammary gland $4.8 \%$, urinary tract $4.8 \%$, respiratory system $4.8 \%$.

As regard the incidence of tumors by age in Cluj County, the following results were obtained: (a) 0-5 years old: skin $46.32 \%$, mammary gland $14.71 \%$, oral cavity $6.62 \%$, orbital region $6.62 \%$, female reproductive system $6.62 \%$, bone and joins $5.15 \%$, alimentary tract $2.94 \%$, lymph nodes $2.21 \%$, urinary tract $2.21 \%$, male reproductive system $2.21 \%$, liver $1.47 \%$, endocrine system 0.74 etc.; (b) 5-10 years old: skin 34.46\%, mammary gland $30.89 \%$, female reproductive system $6.59 \%$, male reproductive system $5.84 \%$, oral cavity $3.77 \%$, bone and joins $3.77 \%$, alimentary tract $3.77 \%$, orbital region $3.01 \%$, respiratory tract
$1.88 \%$, urinary tract $1.51 \%$, lymph nodes $1.13 \%$, liver $0.94 \%$, endocrine system $0.75 \%$ etc.; (c) 10-15 years old: mammary gland $32.49 \%$, skin $27.17 \%$, female reproductive system $7.28 \%$, oral cavity $6.44 \%$, male reproductive system $5.60 \%$, alimentary tract $5.32 \%$, liver $2.24 \%$, orbital region $2.52 \%$, bone and joins $2.52 \%$, respiratory system $1.68 \%$, lymph nodes $1.12 \%$ etc; $(d)>15$ years old: skin $33.33 \%$, mammary gland $27.78 \%$, alimentary tract $11.11 \%$, male reproductive system $11.11 \%$, bone and joins $5.56 \%$, oral cavity $5.56 \%$, female reproductive system $5.56 \%$ etc.

In Cluj County, the incidence of neoplasms in the reproductive system increased with age, somehow similar with the data obtained in Athens. On the other hand, the skin neoplasms rates were similarly distributed in both studies and always in high percentages. In Athens, in younger age groups the incidence rate remained remarkably high (65\%), in middle age groups there was a significant reduction at $36.4 \%$, which continues in elder dogs at $19 \%$, whereas in the study from Cluj County the incidence was constantly over $27 \%$ in all age groups. Regarding the incidence of neoplasms in mammary gland, in both studies the percentages remained low in the younger age group, while approximately doubles in middle age group. In Cluj County, the ratio remained significantly high in elder dogs, whereas in Athens the percentage decreased approximately 3 times from $13.6 \%$ to $4.8 \%$. Another interesting finding was the occurrence of neoplasms in the lymphatic system. In Athens, the incidence rate increases exponentially with age reaching 33.3\% in the elderly, whereas in Cluj County the highest incidence occurred in the younger age group and decreased with age from $2.2 \%$ to $0 \%$.

Bostock (1986a,b) states that the average age of mammary gland cancer development is between 10 and 11 years old, which is confirmed by the results of this study. Another study states that the mean age for lymphoma is $6-9$ years old (Turek et al., 2008; Baba and Cătoi, 2007) which partly coincides with this study.

Concerning the distribution of different types of tumors according to age, the following types of neoplasms appeared in the main age groups in Athens (in brief): (a) $0-6.9$ years: histiocytoma $27.5 \%$, mast cell tumor $17.5 \%$, fibroma $10 \%$, papilloma 5\% etc; (b) 7 - 11.9 years: carcinoma 19.7\%, lymphoma $12.1 \%$, mast cell tumor 9.1\%, 
adenoma $7.6 \%$, fibroma $6.1 \%$, trichoblastoma, Sertoli cell tumor, seminoma, melanoma, hemangiosarcoma, fibrosarcoma, epoulis and adenosarcoma $3.0 \%$ in each etc.; (c) $12-16$ years: carcinoma and hemangiosarcoma $23.8 \%$ in each type of tumor, Sertoli cell tumor, melanoma and lymphoma $9.5 \%$ in each etc.

Concerning the tumor distribution per age groups in Cluj County, the following data were obtained (in short): (a) 0-5 years old: carcinoma $13.76 \%$, histiocytoma $12.84 \%$, melanoma $9.17 \%$, papilloma $9.17 \%$, lymphoma $8.26 \%$, mast cell tumor $8.26 \%$, sticker sarcoma $7.34 \%$, adenoma/ fibroadenoma $6.42 \%$, bone tumor $6.42 \%$, hair follicle tumors $5.5 \%$ etc.; (b) 5-10 years old: carcinoma $34.15 \%$, mast cell tumor $6.65 \%$, melanoma $5.76 \%$, vascular tumors $5.54 \%$, papilloma $5.32 \%$, fibroma/leiomyoma $3.99 \%$, adipose tissue tumors $3.77 \%$ etc.; (c) $10-15$ years old: carcinoma $38.66 \%$, vascular tumors $6.69 \%$, fibroma/leiomyoma 5.95\%, melanoma $5.58 \%$, adenocarcinoma $5.20 \%$, sarcoma $5.20 \%$, adenoma/fibroadenoma $3.72 \%$ etc.; $(d)>15$ years old: vascular tumors $36.36 \%$, carcinoma $27.27 \%$, fibrosarcoma 9.09\%, seminoma 9.09\%, Sertoli cell tumor $9.09 \%$, hair follicle tumors $9.09 \%$ etc.

In both reports the carcinomas had a higher incidence comparing to other tumors. The incidence of histiocytoma in both studies was the highest in young dogs, but in Athens was more than double in incidence (27.5\% vs. $12.8 \%$ ).

The incidence of different types of tumors according to breed in Athens was as follow (the order presented suggests the most commonly affected breeds; presented in brief): Boxer $28.6 \%$ epoulis and mast cell tumor in each, $14.3 \%$ carcinoma, fibroma and lymphoma in each; German shepherd - 44.4\% lymphoma, 11.1\% adenoma, carcinoma, fibrosarcoma, hemangioma and osteoid osteoma in each; Golden Retriever $20 \%$ adenoma, hemangiosarcoma, histiocytoma, lymphoma and melanoma in each; Labrador $28.6 \%$ carcinoma and lymphoma in each, $14.3 \%$ lipoma, mast cell tumor and osteosarcoma in each; Mongrel - 25.8\% carcinoma, 9.7\% seminoma, $6.5 \%$ adenocarcinoma, fibrosarcoma, histiocytoma and mast cell tumor in each; Rottweiler - $28.5 \%$ hemangiosarcoma, $14.3 \%$ seminoma, adenoma, carcinoma, round cell tumor, trichoepithelioma in each; canadian shepherd $-66.6 \%$ fibroma, $33.3 \%$ carcinoma; Shih-tzu - 50\% histiocytoma, 25\% adenoma and hemangiosarcoma in each etc.

The obtained results in this sense were somehow different in Cluj County (the order presented suggests the most commonly affected breeds; presented in brief): Mongrel - carcinoma $41.12 \%$, sticker sarcoma 5.14\%, fibroma/ leiomyoma 5.61\%, mast cell tumors 4.67\%; German shepherd - carcinoma 40.86\%, melanoma $6.45 \%$, vascular cell tumors $6.45 \%$, mast cell tumor - 5.38\%; Spaniel Cocker - carcinoma 46.75\%, adenoma/fibroadenoma $6.49 \%$, melanoma $6.49 \%$, leiomyosarcoma 2.60\%; Caniche carcinoma $32.73 \%$, fibroma/leiomyoma $9.09 \%$, fat tissue tumors $7.27 \%$, Sertoli cell tumor $5.45 \%$, melanoma 5.45\%; Boxer - carcinoma 18.37\%, mast cell tumor $18.37 \%$, papilloma $14.29 \%$, vascular tumors $10.20 \%$, lymphoma $8.16 \%$, melanoma 8.16\%; Rottweiler - carcinoma $24.49 \%$, lymphoma $16.33 \%$, bone tumors $10.20 \%$, vascular tumors $10.20 \%$, mast cell tumors $8.16 \%$; Teckel - carcinoma 36.67\%, adenoma/fibroadenoma $13.33 \%$, papilloma $13.33 \%$, sarcoma $10 \%$; Doberman - carcinoma/adenocarcinoma 35\%, fibrosarcoma 15\%, histiocytoma, melanoma, vascular tumors, adenoma $10 \%$ in each; Schnauzer - carcinoma 31.58\%, melanoma, fibroma/leiomyoma and adenoma $10.53 \%$ in each, epithelioma, sticker sarcoma, hair follicle tumors 5.26\% in each; German Brac - carcinoma/ adenocarcinoma $35 \%$, melanoma $15 \%$, mast cell tumor, fibroma, seminoma $10 \%$ in each.

After comparing the findings obtained in Cluj and Athens regions regarding the incidence of different tumors types according to breed, it was noticed that only few breeds were in common. Furthermore, in the case of similar breeds affected of tumors, little similarities were observed regarding the type of neoplasms. According to Dorn et al. (1968), the rate of breast cancer occurrence in purebred dogs is two times higher than in crossbred dogs of same age. The risk factor to develop cutaneous neoplasms in pure breeds such as German shepherds, miniature poodle and English pointer was two times higher than in mongrels according to Kaldrymidou et al. (2002). Another study states that the highest incidence of mammary tumors occurs in German shepherds (Dobson and Lascelles, 2003). 


\section{CONCLUSION}

The present paper proves that neoplasia is a common morbid entity in pet animals and the incidence increases with age. Tumor cases in household animals are increasing over the years worldwide, aspect that was observed in both Voula-Athens (Greece) and Cluj County (Romania) in the last decade. Several differences were noticed in the two geographic regions assessed. The incidence of mammary gland tumors in Cluj County was two times higher compared to Athens, whereas the occurrence of skin neoplasms was half compared to the records in Greece, where skin neoplasms were the most prevalent tumors. However, the incidence of tumors in the lymphatic system was very low in Cluj (approximately six times less) than in Athens. Concerning the highest tumoral prevalence in various dog breeds, in both reports the most affected were Mongrel and German shepherd breeds. The incidence of cancer in dog fluctuates according to the investigated area, suggesting that determining environmental risk factors may contribute to the neoplastic lesion development.

\section{REFERENCES}

1. American Cancer Society (2011). Global Cancer Facts \& Figures, 2nd Edition, Atlanta, American Cancer Society.

2. Andriopoulou A (2015). The prevalence and incidence of cancer in dogs and cats in a period of ten years $(2005$ - 2014) in Athens, Greece. Department of Pathological Anatomy (Faculty of Veterinary Medicine). University of Agricultural Sciences and Veterinary Medicine ClujNapoca, Romania. Licence Thesis.

3. Baba AI, Cătoi C (2007). Comparative oncology, The Publishing House of Romanian Academy, Bucharest, 34 46.

4. Boffetta P, Nyberg F (2003). Contribution of environmental factors to cancer risk. British Medical Bulletin 68:71-94.

5. Bonnett B, Egenvall NA, Hedhammar A, Olson P (2005). Mortality in over 350000 insured Swedish dogs from 1995-2000: I. Breed, gender, age and causes specific rates. Acta Vet Scand 46:105-120.

6. Bostock DE (1986a). Neoplasms of the skin and subcutaneous tissues in dogs and cats. Br Vet J 142:1-19.

7. Bostock DE (1986b). Canine and feline mammary neoplasm. Br Vet J 142:506-515.

8. Brearley MJ (1989). Mammary gland tumors in the dog. In Practice 11:248-253.
9. Cohen AJ (2000). Outdoor air pollution and lung cancer. Environ Health Perspect 108(4):743-750.

10. Dobson JM, Duncan B, Lascelles X (2003). BSAVA Manual of canine and feline oncology. Second edition, British Small Animal Veterinary Association, 234-242.

11. Dorn CR, Priester WA (1987). Epidemiology. In: Theilen GH, Madewell BR (Eds). Veterinary Cancer Medicine, 2nd ed, JB Lippincott, Philadelphia, 14-32.

12. Dorn CR, Taylor D, Schneider R, Hibbard HH, Klauber MR (1968). Survey of animal neoplasms in Alameda and Contra Costa Counties, California. II. Cancer morbidity in dogs and cats from Alameda County. J Natl Cancer Inst 40:307-318.

13. Hemminki K, Pershagen G (1994). Cancer risk of air pollution: epidemiological evidence. Environ Health Perspect 102(4):187-192.

14. Kaldrymidou H, Leontides L, Koutinas AF, Saridomichelakis MN, Karayannopoulou M (2002). Prevalence, distribution and factors associated with the presence and the potential for malignancy of cutaneous neoplasms in 174 dogs admitted to a clinic in Northern Greece. J Vet Med 49:8791.

15. Kelsey J, Moore A, Glickman L (1998). Epidemiologic Studies of Risk Factors for Cancer in Pet Dogs. Epidemiology reviews 20(2):204-217.

16. Pershagen G, Simonato L (1993). Epidemiological evidence on outdoor air pollution and cancer. In : Tomatis L (ed). Indoor and Outdoor Air Pollution and Human Cancer, Springer-Verlag, Berlin, 135-148.

17. Petrauskaite R, Pershagen G, Gurevicius R (2002). Lung cancer near an industrial site in Lithuania with major emissions of airway irritants. Int J Cancer 99:106-111.

18. Popa A (2014). Incidence and prevalence of tumors in dogs and cats in a period of ten years (2004-2013) in Cluj County (Romania). Department of Pathological Anatomy (FVM Cluj-Napoca, Romania), University of Agricultural Sciences and Veterinary Medicine Cluj- Napoca, Romania. Licence Thesis, 26-58.

19. Rungsipipat A, Sunyasootcharee B, Ousawaphlangchai L, Sailasuta A, Thanawongnuwech R, Teankum K (2003). Neoplasms of dogs in Bangkok Thailand. J Vet Med 33:5966.

20. Siegel R, Naishadham D, Jemal A (2013). Cancer statistics. Cancer J Clin 63:11-30.

21. Turek MM, Saba C, Paoloni CM, Argyle JD (2008). Canine Lymphoma and Leukemia. In : JD Argyle \& MJ Brearley \& MM Turek (Eds). Decision Making in Small Animal Oncology, Wiley-Blackwell, 171-196.

22. Vascellari M, Baioni E, Ru G, Carminato A, Mutinelli F (2009). Animal tumor registry of two provinces in northern Italy: incidence of spontaneous tumors in dogs and cats. BioMed Central Ltd. BMC Vet Res 5:39. 